\title{
A clinical trial of single dose rectal and oral administration of diazepam for the prevention of serial seizures in adult epileptic patients
}

\author{
NM MILLIGAN, S DHILLON, A GRIFFITHS, J OXLEY, A RICHENS \\ From the Institute of Neurology, London, Chalfont Centre for Epilepsy, Chalfont St Peter, Bucks, and the \\ Department of Pharmacology and Therapeutics, Welsh National School of Medicine, Heath Park, Cardiff, \\ $U K$
}

SUMMARY The clinical anticonvulsant efficacy of single dose rectal and oral administration of diazepam $20 \mathrm{mg}$ was examined in two double-blind placebo-controlled trials in adult epileptic patients. All subjects suffered from drug resistant epilepsy and frequently experienced serial seizures. Diazepam was administered rectally as a new experimental suppository formulation immediately after a seizure and was highly effective in preventing recurrent fits within a $24 \mathrm{~h}$ observation period $(p<0 \cdot 001)$. Pharmacokinetic studies revealed a wide range of serum diazepam concentrations $60 \mathrm{~min}$ after administration of the suppository (mean serum diazepam level $190 \pm 73$ (SD) $\mathrm{ng} / \mathrm{ml}$ ). In a similar study oral administration of diazepam $20 \mathrm{mg}$ significantly reduced the incidence of serial seizures compared with a placebo $(p<0.01)$ and the mean 60 min serum diazepam level was $273 \pm 190(\mathrm{SD}) \mathrm{ng} / \mathrm{ml}$.

A proportion of patients with epilepsy suffer from serial seizures or fits in clusters, that is, two or more fits in quick succession excluding status epilepticus. This condition leads to an increased morbidity from epileptic seizures and may herald true status epilepticus. ${ }^{12}$ Consequently, serial seizures should be controlled as soon as possible to prevent the development of more serious complications. Ideally, this requires an agent which is both effective and easily administered without the skills required for parenteral administration. Since many patients remain unconscious after a fit oral administration is often impractical and may also be ineffective although this has never been examined in a clinical trial. Recently, rectal administration of diazepam solution in children has been shown to be clinically effective in the acute treatment of seizures ${ }^{3}$ and in the prevention of recurrent febrile convulsions. ${ }^{4}$ When given rectally in adults diazepam solution significantly reduces the frequency of interictal spikes in the EEG but the clinical value of such treatment is uncertain.

Address for reprint requests: Dr NM Milligan, Department of Neurology, University Hospital of Wales, Cardiff CF4 4XW, UK.

Received 30 June 1983 and in revised form 10 September 1983 Accepted 24 September 1983
Diazepam suppositories provide a more convenient mode of drug administration but the results from previous pharmacokinetic studies have been disappointing and this may be related to the formulations used. ${ }^{67}$ Consequently the use of these suppositories in the treatment of adult patients with epilepsy has never been systematically examined. A new "solid solution" diazepam suppository was specifically designed to promote rapid release of the drug. This preparation has been shown to have significantly better absorption characteristics compared with the commercially available wax-based Valium (Roche) suppository ${ }^{7}$ and was highly effective in terminating recurrent absence status in a single case study. ${ }^{8}$ Since intermittent rectal instillation of diazepam solution in children is known to be an effective prophylactic agent against recurrent febrile convulsions, the prevention of serial seizures in adults provided a suitable model on which to examine further the clinical efficacy of the new diazepam suppository. In addition, since the absorption profiles and bio-availability of diazepam administered orally and rectally are similar a second study was undertaken to examine the clinical efficacy of intermittent oral administration of diazepam using the same experimental model. 


\section{Methods}

Diazepam suppository study

Twenty-two patients, mean age $30 \cdot 2$ years (range $18-50$ ) and resident at the Chalfont Centre for Epilepsy, gave their signed informed consent to take part in this study. All patients suffered from severe epilepsy uncontrolled by existing medication (carbamazepine, phenytoin, phenobarbitone, primidone, and sodium valproate either singly or in combination). The study was confined to subjects known to have fits in clusters, that is, two or more fits in $24 \mathrm{~h}$ (generalised tonic-clonic and easily recognised partial seizures) excluding status epilepticus. Treatment consisted of the new "solid solution" diazepam suppository $20 \mathrm{mg}$ and a matched placebo supplied by Dr B Winsley, John Radcliffe Hospital, Oxford. A dose of $20 \mathrm{mg}$ was used because previous pharmacokinetic studies have shown that serum levels above the suggested minimum effective serum concentration of $150 \mathrm{ng} / \mathrm{ml}$ were best achieved with this dosage. 5 Suppositories were dispensed by the hospital pharmacy in code numbered containers. Each patient had his own labelled box containing one diazepam and one placebo suppository. When two suppositories had been used in a given subject they were replaced by another pair (one active, one placebo) for use in the same patient on subsequent occasions to ensure that those patients studied relatively infrequently had the opportunity of receiving both treatments and the first treatment of each pair was allocated randomly. Administration of suppositories and patient assessments were conducted by the attendant staff under double-blind conditions.

In a given patient, a single suppository was administered as soon as possible after a fit. The time and description of the fit were documented on record sheets provided together with the code number of the treatment given. Patients remained under observation for the next $24 \mathrm{~h}$ and all fits occurring during this time were accurately recorded. In the majority of cases a minimum wash-out period of $48 \mathrm{~h}$ was allowed between treatments although on a few occasions patients were studied more frequently when the opportunity presented (wash-out period 24-48 h). The nursing or medical staff were permitted to administer alternative treatment if seizures occurred frequently after administration of the suppository.

A single blood specimen $(7 \mathrm{ml})$ was taken $60 \mathrm{~min}$ after administration of the suppository. When this was impractical samples were withdrawn at a more convenient time. These were subsequently analysed by gas chromatography for serum diazepam concentrations. Since diazepam was given intermittently and its use was intended as an adjunct to regular medication no attempt was made to hold constant the dosage of routine antiepileptic drug therapy and this could be altered at any time if clinically indicated. Assessments were based on the incidence of recurrent seizures recorded by the nursing staff in the $24 \mathrm{~h}$ period immediately following treatment. When multiple fits recurred these were counted as one recurrence. The final analysis was confined to those recurrent fits in which the nature of the treatment given was confirmed by serum drug analysis and the Mantel-Haenszel Chi-squared test was used for statistical analysis. This test has the advantage over the more widely used Chi-squared test with Yates' correction of stratifying the data by subject, thus allowing each patient to be used as his own control' ${ }^{9}$ and gives a $\chi^{2}$ value which adequately takes into account the multiple cross-over structure of the trial.

\section{Oral diazepam study}

Eighteen adult epileptic patients, mean age 38 years (range 21-60) and resident at the Chalfont Centre for Epilepsy, consented to take part in this study. All patients suffered from severe epilepsy with mixed seizure types and all subjects were prone to serial epilepsy or fits in clusters from which they were known to make a rapid recovery. This permitted treatment to be administered orally. Treatment consisted of diazepam tablets $20 \mathrm{mg}$ and a matched placebo supplied by Roche Ltd. The study was conducted double-blind and the procedure was identical to that used in the previous study with the exception that treatment was administered orally. It was usually possible to administer tablets within $30 \mathrm{~min}$ of a fit and special care was taken to ensure that tablets were properly swallowed. The effect of treatment was based on the incidence of recurrent fits in the $24 \mathrm{~h}$ observation period following treatment and the Mantel-Haenzsel Chi-squared test was used for statistical analysis.

\section{Results}

\section{Diazepam suppository study}

No adverse effects from the new suppository formulation were observed in any subject and no patient developed status epilepticus. Only two patients underwent a change in routine antiepileptic drug therapy during the trial. A total of 81 suppositories were administered and blood specimens were taken in 66 instances. The remaining 15 treatments were excluded from data analysis because no blood specimens were obtained. We have assumed that the presence of diazepam in blood indicated that active treatment was administered and the absence of diazepam implied that a placebo suppository was given. Analysis of the 66 specimens revealed that 35 samples contained diazepam and 31 were diazepam free (that is, placebo had been administered). There were five discrepancies between the results from serum drug analysis and the Pharmacist's code indicating a mis-match between intention and practise on these occasions. Of the 22 patients in the trial, 17 had a recurrent fit after treatment on at least one occasion and the blood specimens from these 17 patients accounted for 58 of the 66 confirmed treatments (diazepam 31, placebo 27). Fits recurred on $6 / 31$ occasions after diazepam compared with $21 / 27$ occasions after placebo $(p<0 \cdot 001)$. The eight treatments from the five patients who did not experience recurrent fits were not included in the analysis. Details of the frequency and distribution of recurrent fits among patients and the seizure type before and after treatment are given in table 1 . It 
Table 1 Frequency and distribution of recurrent fits in the $24 \mathrm{~h}$ period following administration of diazepam and placebo suppositories. When multiple fits recurred these were counted as one recurrence in the final analysis. Fits recurred on only $6 / 31$ occasions after diazepam compared with $21 / 27$ occasions following placebo (Mantel-Haenszel $\chi^{2}=18 \cdot 50 ; d f=1$; $p<0.001)$

\begin{tabular}{|c|c|c|c|c|c|c|c|}
\hline \multirow[t]{2}{*}{ Subject } & \multicolumn{2}{|c|}{ Number of treatments } & \multirow{2}{*}{$\begin{array}{l}\text { No. of } \\
\text { recurrent } \\
\text { fus }\end{array}$} & \multicolumn{2}{|c|}{ Time to fut $(h)$} & \multicolumn{2}{|l|}{ Fit type } \\
\hline & $D Z P$ & Placebo & & $D Z P$ & Placebo & Before & After \\
\hline $\begin{array}{l}\text { G.B. } \\
\text { G.B. } \\
\text { G.B. } \\
\text { K.B. } \\
\text { D.C. } \\
\text { M.H. } \\
\text { M.H. } \\
\text { M.H. } \\
\text { M.H. } \\
\text { M.H. } \\
\text { M.H. } \\
\text { S.H. } \\
\text { S.Ho. } \\
\text { B.L. } \\
\text { K.L. } \\
\text { K.L. } \\
\text { M.M. } \\
\text { M.M. } \\
\text { D.P. } \\
\text { M.P. } \\
\text { A.R. } \\
\text { E.S. } \\
\text { J.S. } \\
\text { H.V. } \\
\text { H.V. } \\
\text { R.W. } \\
\text { R.Wo. }\end{array}$ & $\begin{array}{l}1 \\
0 \\
1 \\
5 \\
3 \\
2 \\
2 \\
0 \\
2 \\
0 \\
2 \\
2 \\
1\end{array}$ & $\begin{array}{l}2 \\
1 \\
1 \\
2 \\
1 \\
2\end{array}$ & $\begin{array}{l}1 \\
1 \\
1 \\
2 \\
2 \\
1 \\
1 \\
1 \\
1 \\
1 \\
2\end{array}$ & $\begin{array}{l}1 ; 4^{*} \\
6 \cdot 5 \\
\\
8 \cdot 5 \\
14 \cdot 5 \\
0 \cdot 75 ; 7\end{array}$ & $\begin{array}{l}1 ; 3 \\
1 ; 22 \\
1 \\
1 \cdot 25 \\
2 \\
5 \\
0 \cdot 1 \\
3 \\
23 \cdot 5 \\
1 \cdot 5 \\
6 \\
1 \cdot 5 \\
4 \\
0 \cdot 5 ; 16 \\
5 ; 10 \cdot 5 \\
16 \\
0 \cdot 5 ; 23 \\
24\end{array}$ & $\begin{array}{l}\text { CP } \\
\text { CP } \\
\text { CP } \\
\text { T } \\
\text { CP } \\
\text { Serial CP } \\
\text { CP } \\
\text { TC } \\
\text { CP } \\
\text { TC } \\
\text { CP } \\
\text { TC } \\
\text { T } \\
\text { TC } \\
\text { CP } \\
\text { TC } \\
\text { CP } \\
\text { TC } \\
\text { TC } \\
\text { TC } \\
\text { TC } \\
\text { TC } \\
\text { TC } \\
\text { TC } \\
\text { TC } \\
\text { T } \\
\text { Ad }\end{array}$ & $\begin{array}{l}\text { CP } \times 2 \\
\text { TC } \\
\text { CP } \times 2 \\
\text { T } \\
\text { CP } \\
\text { CP } \\
\text { Serial CP } \\
\text { CP } \times 2 \\
\text { CP } \times 2 \\
\text { CP } \times 2 \\
\text { Serial CP } \\
\text { TC } \\
\text { T } \\
\text { CP } \times 2 \\
\text { CP } \\
\text { CP } \\
\text { TC } \\
\text { TC } \\
\text { TC } \\
\text { TC } \\
\text { TC } \\
\text { TC } \\
\text { TC } \times 2 \\
\text { TC } \times 2 \\
\text { TC } \\
\text { T } \times 2 \\
\text { Ad }\end{array}$ \\
\hline $\begin{array}{l}\text { DZP: } \\
\text { CP: } \\
\text { TC: }\end{array}$ & $\begin{array}{l}\text { urtial se } \\
\text { seizur }\end{array}$ & & & $\begin{array}{l}\text { tonic se } \\
\text { adversiv } \\
\text { bowels }\end{array}$ & minute & pository ad & stration \\
\hline
\end{tabular}

can be seen that, following placebo recurrent fits were identical in type to the fit immediately before administration of the suppository on 18 of the 21 occasions when fits recurred.

Absorption of diazepam from the rectum was highly variable as judged by a wide range of diazepam concentrations in blood samples obtained at $60 \mathrm{~min}$ (mean $190 \pm 73(\mathrm{SD}) \mathrm{ng} / \mathrm{ml}$; range 40 $340 \mathrm{ng} / \mathrm{ml} ; \mathrm{n}=25)$. In one subject (BL) seizures recurred within 20 minutes of diazepam administration $(60 \mathrm{~min}$ serum level $170 \mathrm{ng} / \mathrm{ml})$ and in another (GB) the suppository was not retained $(60 \mathrm{~min}$ serum level $50 \mathrm{ng} / \mathrm{ml}$ ).

\section{Oral diazepam study}

No adverse effects were encountered in any subject and only one patient underwent a change in routine antiepileptic drug therapy during the trial. A total of 95 treatments were administered of which 85 were confirmed by serum drug analysis (diazepam 46 , placebo 39). There were nine discrepancies between the results from drug analysis and the Pharmacist's code. Of the 18 patients in the trial, 12 experienced recurrent fits and the blood specimens from these 12 patients accounted for 73 of the 85 confirmed treatments (diazepam 38, placebo 35). Fits recurred on 11/38 occasions after treatment with diazepam compared with 21/35 occasions after placebo $(p<0 \cdot 01)$. The 12 treatments in the six patients who did not experience recurrent fits were not included in the analysis. Table 2 shows the number and distribution of fits before and after treatment. It can be seen that pre-treatment and recurrent fits were identical in type on all of the 11 occasions when fits recurred after diazepam compared with 18 of 21 such occasions after placebo.

The mean 60 min serum diazepam concentration was $273 \pm 190(\mathrm{SD}) \mathrm{ng} / \mathrm{ml}$ (range $15-570 \mathrm{ng} / \mathrm{ml}$; $\mathrm{n}=30$ ) and the 60 min serum concentrations in the 11 treatment failures after diazepam ranged from $38-570 \mathrm{ng} / \mathrm{ml} \quad$ (mean $292 \pm 203$ (SD) $\mathrm{ng} / \mathrm{ml}$; $\mathrm{n}=10)$.

\section{Discussion}

With the exception of an isolated case report, ${ }^{8}$ single dose rectal or oral administration of diazepam has never previously been shown to have anticonvulsant activity in adult epileptic patients. These results show that intermittent administration of diazepam 
Table 2 Frequency and distribution of recurrent fits following oral administration of diazepam and placebo. Fits recurred on 11/38 occasions after diazepam compared with 21/35 occasions after placebo (Mantel-Haenszel $\chi^{2}=8.44 ; d f=1$; $p<0.01)$

\begin{tabular}{|c|c|c|c|c|c|c|c|}
\hline \multirow[t]{2}{*}{ Subjects } & \multicolumn{2}{|c|}{ Number of treatments } & \multirow{2}{*}{$\begin{array}{l}\text { No. of } \\
\text { recurrent } \\
\text { fits }\end{array}$} & \multicolumn{2}{|c|}{ Time to fit (h) } & \multicolumn{2}{|l|}{ Fit type } \\
\hline & $D Z P$ & Placebo & & $D Z P$ & Placebo & Before & After \\
\hline $\begin{array}{l}\text { F.B. } \\
\text { F.B. } \\
\text { F.B. } \\
\text { F.B. } \\
\text { F.B. } \\
\text { G.B. } \\
\text { G.B. } \\
\text { K.D. } \\
\text { K.D. } \\
\text { L.F. } \\
\text { L.F. } \\
\text { L.F. } \\
\text { A.H. } \\
\text { S.K. } \\
\text { R.M. } \\
\text { R.M. } \\
\text { C.P. } \\
\text { C.P. } \\
\text { M.P. } \\
\text { M.P. } \\
\text { M.P. } \\
\text { M.P. } \\
\text { M.P. } \\
\text { M.R. } \\
\text { M.R. } \\
\text { M.R. } \\
\text { M.R. } \\
\text { E.S. } \\
\text { E.S. } \\
\text { R.W. } \\
\text { R.W. } \\
\text { R.W. }\end{array}$ & $\begin{array}{l}2 \\
4 \\
2\end{array}$ & $\begin{array}{l}2 \\
0 \\
5 \\
4 \\
5\end{array}$ & $\begin{array}{l}1 \\
1 \\
2 \\
2 \\
5\end{array}$ & $\begin{array}{l} \\
21 ; 22 \\
0 \cdot 1 \\
16 \\
12 ; 20 \\
0 \cdot 2 \\
0 \cdot 1\end{array}$ & $\begin{array}{l}3 \cdot 5 \\
5 \\
1 \\
23 \\
0 \cdot 5 \\
15 ; 17 \\
0 \cdot 5 ; 17 \\
7 \\
5 \\
\\
0 \cdot 5 ; 1 \\
18 \\
19 \\
4 ; 5 \\
20 \\
3 \cdot 5\end{array}$ & $\begin{array}{l}\text { CP } \\
\text { CP } \\
\text { CP } \\
\text { CP } \\
\text { CP } \\
\text { CP } \\
\text { CP } \\
\text { TC } \\
\text { TC } \\
\text { At } \\
\text { Serial CP } \\
\text { At } \\
\text { Ad } \\
\text { Serial Ad } \\
\text { TC } \\
\text { CP } \\
\text { CP } \\
\text { CP } \\
\text { CP } \\
\text { Serial CP } \\
\text { CP } \\
\text { At } \\
\text { Serial CP } \\
\text { Serial CP } \\
\text { CP } \\
\text { CP } \\
\text { CP } \\
\text { CP } \\
\text { TC } \\
\text { CP } \\
\text { CP } \\
\text { CP }\end{array}$ & $\begin{array}{l}\text { CP } \times 2 \\
\text { CP } \\
\text { CP } \\
\text { CP } \times 2 \\
\text { CP, TC } \\
\text { CP } \\
\text { CP } \times 2 \\
\text { TC } \times 2 \\
\text { TC } \\
\text { At } \times 2 \\
\text { CP } \\
\text { At } \times 2 \\
\text { Ad } \\
\text { Ad } \\
\text { TC } \\
\text { TC } \\
\text { Serial CP } \\
\text { CP } \\
C P \\
\text { CP } \times 2 \\
C P \times 2 \\
\text { Serial CP } \\
\text { Serial CP } \\
\text { Serial CP } \\
C P \times 2 \\
C P \\
\text { Serial CP } \\
\text { TC } \times 2 \\
\text { TC } \\
\text { Serial CP } \\
C P \\
C P \\
\text { CP }\end{array}$ \\
\hline
\end{tabular}

$20 \mathrm{mg}$ either orally or rectally as a rapid release suppository formulation was significantly better than placebo in preventing serial seizures in patients prone to fits in clusters. Serum concentrations of $\mathrm{N}$-desmethyldiazepam were measured in the first study and found to be negligible indicating that these results are unlikely to be due to accumulation of active metabolites. On one of the six occasions when fits recurred after the diazepam suppository the preparation was not retained and on another fits recurred within 20 min of drug administration when maximum absorption would not have been achieved. Similarly, on two occasions after oral diazepam fits recurred within $10 \mathrm{~min}$ of the tablets being swallowed. The striking effectiveness of diazepam in preventing serial seizures in this sample of epileptic patients was surprising since all patients suffered from severe epilepsy resistant to conventional antiepileptic drugs. These results are therefore very encouraging. It was not possible to determine from these results which type of fit was likely to best respond to diazepam as all patients included in the trial suffered from mixed seizure types. However, the striking similarity of the fits before and after treatment in both studies suggests that most seizure types including tonic-clonic fits. Although the suppository appeared more effective than oral diazepam in preventing serial seizures this cannot be seen as evidence of superiority of one preparation over another in view of the small sample size and in view of the relatively small difference in the frequency of recurrent fits after the suppository (19\%) compared with that after oral diazepam (29\%).

The main objective of obtaining blood specimens in this study was to verify the nature of the treatment given and in view of the lack of a strict sampling time strategy the significance of the wide range of serum diazepam concentrations achieved cannot be stressed. However, previous pharmacokinetic studies in adult epileptic patients have shown considerable variation in serum levels achieved between subjects following oral administration of diazepam and when diazepam is given rectally in solution or as the new rapid release suppository. ${ }^{57}$ Successful prophylaxis against serial seizures in this study almost certainly occurred with an equally wide range 
of absorption profiles. Whilst delay in drug absorption may influence therapeutic efficacy in patients who are continually fitting this did not affect the overall results from the present investigation but maximum protection against recurrence of seizures may not occur until 30-60 min after administration of diazepam and patients may still be at risk during this time. Prior stomach and bowel preparation may have resulted in more consistent absorption but this will not be feasible in clinical practice. Rectal instillation of diazepam solution through a catheter attached to a syringe results in more rapid drug absorption ${ }^{58}$ and will reduce the period of risk from early recurrence of fits but this method of administration is cumbersome compared with a suppository and may not be practicable in the home environment. Special applicators (Stesolid, Weddel) have been utilised to squirt a fixed dose of diazepam into the rectum but these result in low bioavailability which may occur because of incomplete emptying or sucking back of part of the dose on removal of the applicator from the rectum. ${ }^{10}$ Moreover, Stesolid applicators instil only $10 \mathrm{mg}$ of diazepam which may be insufficient to achieve an anticonvulsant effect. Diazepam suppositories provide the most convenient dosage form for rectal use and the new rapid release formulation used in this investigation has given encouraging results. A similar study in children investigating the use of these suppositories for the prevention of recurrent febrile convulsions would be a logical extension of work already done. Since continuous prophylaxis is unnecessary and unsatisfactory in many children with febrile convulsions $^{11-13}$ the use of this preparation given intermittently as a prophylactic agent during febrile periods is an attractive alternative therapy and this should be examined further. It should be emphasised, however, that this preparation is still an experimental formulation and may not become commercially available. Although concern has recently been expressed regarding possible solvent-induced mucosal damage following rectal administration of diazepam solution no such problems were encountered in this clinical trial. Moreover, extensive clinical use of diazepam solution given per rectum in Scandinavia has not revealed any such sequelae and more formal studies in rabbits suggest that this is not a problem. ${ }^{14}$ Whilst the commercial development of a new suppository formulation requires detailed and expensive toxicological studies to ensure the safety of the preparation, the results from this and other studies are very encouraging and fully justify our recommendation that this agent be further assessed by the pharmaceutical industry particularly as the currently available preparations are so poorly absorbed. The potential clinical uses of intermittent therapy with diazepam provide additional means for the management of patients with intractable epilepsy which hitherto have not been fully recognised. When given as an adjunct to regular medication it can be used at times of poor fit control which often occur during periods of drug adjustment or at the menses and these results suggest that diazepam could be given orally if practical and rectally if not.

Whilst this treatment may not stop fits completely it will reduce the morbidity from recurrent fits and it may also prevent the development of status epilepticus. In addition, this treatment may lessen the number of hospital admissions from serial seizures which frequently lead to well meaning but often fruitless attempts at reconstructing patients' drug regimens. Furthermore, intermittent diazepam therapy may allow complex drug regimens to be simplified and thus reduce the hazards from continued polypharmacy. It should be noted, however, that prolonged sedation is the most common adverse effect from diazepam and this may be unacceptable to patients who recover rapidly from their seizures if this treatment is promoted on a wide scale. A drug equivalent in antiepileptic potency to diazepam but without the sedative effects of this drug would clearly be a more suitable prophylactic agent for use against serial seizures. The new 1,5 benzodiazepine, clobazam (Frisium, Hoechst) may fulfil these requirements since this drug impairs psychomotor performance less than diazepam ${ }^{15}$ and has recently been shown to have antiepileptic properties after acute administration in animals. ${ }^{16} \mathrm{~A}$ model based on the prevention of serial seizures in man has never previously been used for the clinical assessment of an antiepileptic drug but the possibility of using such a model for the assessment of a new drug for example clobazam given, say, as a single dose immediately after a fit is a most attractive one and this should be examined further.

This work was supported by a grant from The Thorn Epilepsy Research Trust. The authors are grateful to Dr B Winsley for supply of diazepam and placebo suppositories, to Roche Ltd for supply of diazepam and placebo tablets and to Dr $R$ Newcombe for statistical advice. Secretarial help from Mrs EM Long is gratefully acknowledged.

\section{References}

${ }^{1}$ Richens A. Clinical pharmacology and medical treatment. In: Laidlaw J, Richens A, eds. A Textbook of Epilepsy. Edinburgh: Churchill Livingstone, 1982: 339.

${ }^{2}$ Marsden CD, Reynolds EH. Neurology. In: Laidlaw J, Richens A, eds. A Textbook of Epilepsy. Edinburgh: Churchill Livingstone, 1982:107. 
${ }^{3}$ Knudsen FU. Rectal administration of diazepam in solution in the acute treatment of convulsions in infants and children. Arch Dis Child 1979;54:855-7.

${ }^{4}$ Knudsen FU, Vestermark S. Prophylactic diazepam or phenobarbitone in febrile convulsions: a prospective controlled study. Arch Dis Child 1978;53:660-3.

${ }^{5}$ Milligan N, Dhillon S, Oxley I, Richens A. Absorption of diazepam from the rectum and its effect on the frequency of inter-ictal spikes in the EEG. Epilepsia 1982;23:323-31.

${ }^{6}$ Moolenaar F, Bakker S, Visser J, Huizinga T. Biopharmaceutics of rectal administration of drugs in man IX. Comparative biopharmaceutics of diazepam after single rectal, oral, intramuscular and intravenous administration in man. Int $J$ Pharmaceutics 1980;5:127-37.

${ }^{7}$ Dhillon S, Oxley J, Richens A. Bioavailability of diazepam after intravenous, oral and rectal administration in adult epileptic patients. Br J Clin Pharmacol 1982;13:427-32.

${ }^{8}$ Milligan N, Dhillon S, Oxley J, Richens A. Rectal diazepam in the treatment of absence status: a pharmacodynamic study. $J$ Neurol Neurosurg and Psychiatry 1981;44:914-7.

${ }^{9}$ Mantel N, Haenszel W. Statistical aspects of the analysis of data from retrospective studies of disease. $\mathrm{J} \mathrm{Nat}$
Cancer Inst 1959;22:719-49.

${ }^{10}$ Magnussen I, Oxlund HRW, Alsbirk KE, Arnold E. Absorption of diazepam in man following rectal and parenteral administration. Acta Pharmacol Toxicol 1979;45:87-90.

" Heckmatt JZ, Houston AB, Clow DJ, et al. Failure of phenobarbitone to prevent febrile convulsions. $\mathrm{Br}$ Med J 1976;1:559-61.

12 Thorn I. A controlled study of prophylactic long term treatment of febrile convulsions with phenobarbitone. Acta Neurol Scand (Suppl) 1975;60:67-73.

${ }^{13}$ Melchior JC, Buchthal F, Lennox-Buchthal M. The effectiveness of diphenylhydantoin in preventing febrile convulsions at the age of greatest risk, under 3 years. Epilepsia 1971;12:55-62.

${ }^{14}$ Munthe-Kass AW. Rectal administration of diazepam: theoretical basis and clinical experience. In: Johannessen SI, et al. eds. Antiepileptic Drug Therapy: Advances in Drug Monitoring. New York: Raven Press, 381-90.

${ }^{15}$ Hindmarch I. Some aspects of the effects of clobazam on human psychomotor performance. $\mathrm{Br} J$ Clin Pharmacol 1979;7 (Suppl 1):77-82.

${ }^{16}$ Meldrum BS, Chapman AG, Horton RW. Clobazam: Anticonvulsant action in animal models of epilepsy. Br J Clin Pharmacol 1979;7 (Suppl 1):59-60. 